\title{
Structure determination from powder X-ray diffraction data augmented by complementary techniques
}

\author{
Kenneth D. M. Harris \\ School of Chemistry, Cardiff University, Cardiff CF10 3AT, Wales, United Kingdom \\ HarrisKDM@cardiff.ac.uk
}

Structure determination of organic materials directly from powder X-ray diffraction (XRD) data [1,2] is now carried out extensively by researchers in both academia and industry. Most research in this field uses the direct-space strategy for structure solution $[3,4]$ followed by Rietveld refinement. Although the structure determination process is generally carried out solely using powder XRD data, significant advantages may be gained by augmenting the process of structure determination from powder XRD data by utilizing information obtained from other experimental and computational techniques. Such multi-technique approaches are particularly advantageous in tackling complex and challenging structure determination problems, both by providing independent information that may be used directly to facilitate the structure determination process and by allowing robust validation of the final structure obtained in the Rietveld refinement. The lecture will focus on the use of solid-state NMR spectroscopy and periodic DFT-D calculations to augment the process of structure determination of organic materials from powder XRD data [5-11]. The lecture will present several case studies from recent research, including several examples of polymorphic systems and pharmaceutical materials. Recent examples exploiting the complementary advantages of 3D electron diffraction data and powder XRD data within the structure determination process will also be presented.

1. Harris, K. D. M., Tremayne, M. \& Kariuki, B. M. (2001) Angew. Chemie Int. Ed. 40, 1626.

2. Harris, K. D. M. (2012) Top. Curr. Chem. 315, 133.

3. Harris, K. D. M., Tremayne, M., Lightfoot, P. \& Bruce, P. G. (1994) J. Am. Chem. Soc. 116, 3543.

4. Kariuki, B. M., Serrano-González, H., Johnston, R. L. \& Harris, K. D. M. (1997) Chem. Phys. Lett. 280, 189.

5. Dudenko, D. V., Williams, P. A., Hughes, C. E., Antzutkin, O. N., Velaga, S. P., Brown, S. P. \& Harris, K. D. M. (2013) J. Phys. Chem. C $117,12258$.

6. Williams, P. A., Hughes, C. E. \& Harris, K. D. M. (2015) Angew. Chemie Int. Ed. 54, 3973.

7. Watts, A. E., Maruyoshi, K., Hughes, C. E., Brown, S. P. \& Harris, K. D. M. (2016) Cryst. Growth Des. 16, 1798.

8. Hughes, C. E., Reddy, G. N. M., Masiero, S., Brown, S. P., Williams, P. A. \& Harris, K. D. M. (2017) Chem. Sci. 8, 3971.

9. Hughes, C. E., Boughdiri, I., Bouakkaz, C., Williams, P. A. \& Harris, K. D. M. (2018) Cryst. Growth Des. 18, 42.

10. Al Rahal, O., Hughes, C. E., Williams, P. A., Logsdail, A. J., Diskin-Posner, Y. \& Harris, K. D. M. (2019) Angew. Chemie Int. Ed. 58, 18788.

11. Al Rahal, O., Williams, P. A., Hughes, C. E., Kariuki, B. M. \& Harris, K. D. M. (2021) Cryst. Growth Des. $21,2498$.

Keywords: Powder X-ray Diffraction; Structure Determination; Solid-state NMR; DFT Calculations; 3D Electron Diffraction 